\title{
Evaluation of Right Ventricle Functions Might Be a Method for Early Recognizing Cardiotoxicity in Hematopoietic Stem Cell Transplantation
}

\author{
Fatih Tekiner, Cennet Yildiz', Sebnem Izmir Guner ${ }^{2}$ \\ Altınbaş University, Medical Park Bahcelievler Hospital, ${ }^{1}$ Kavram Vocational School, ${ }^{2}$ Memorial Şişli Hospital, Istanbul, Turkey \\ ORCID: \\ Fatih Tekiner: 0000-0003-0146-5830 \\ Cennet Yildiz: 0000-0003-2456-3206 \\ Sebnem Izmir Guner: 0000-0002-6326-9424
}

\section{Abstract}

Objective: We aimed to investigate whether right ventricular (RV) functional assessment by echocardiography may help in the early diagnosis of hematopoietic stem cell transplantation (HSCT)-induced cardiotoxicity. Methods: Our study population comprised 41 autologous multiple myeloma, 25 autologous Hodgkin's lymphoma, 20 autologous and 19 allogeneic non-Hodgkin's lymphoma, 10 allogeneic acute myeloid leukemia, 12 allogeneic myelodysplastic syndrome, and 10 allogeneic aplastic anemia patients. Conventional two-dimensional echocardiographic and tissue Doppler imaging examination of the patients were performed before and after HSCT. Post-HSCT values were compared with pre-HSCT values. Results: The mean age of the patients was $46.51 \pm 16.24$ years, and the mean hospital length of stay and the mean leukocyte engraftment time were 30 and 11 days, respectively. The number of female and male patients was 60 (43.8\%) and $77(56.2 \%)$, respectively. There were no significant differences in echocardiographic parameters including left ventricular end-diastolic diameter, left ventricular end-systolic diameter, left ventricular ejection fraction, RV, right atrium, pulmonary artery pressure, pulmonary velocity, Pulmonary acceleration time (PAT)/ pulmonary ejection time (PET), PAT, E, A wave velocity, E/A ratio, and DT and A' velocity before and after HSCT. Compared to pretransplant values, posttransplant values of the E' velocity, S' velocity, E'/A' ratio, and tricuspid annular plane systolic excursion showed a statistically significant decrease $(0.15 \pm 0.34$ vs. $0.12 \pm 0.04, P=0.032 ; 0.19 \pm 0.06$ vs. $0.09 \pm 0.04, P<0.001 ; 0.90 \pm 0.40$ vs. $0.72 \pm 0.37, P=0.012$; and $2.30 \pm 0.39$ vs. $1.63 \pm 0.30, P<0.001$, respectively). Conclusion: Assessment of RV functions might be useful for the prediction of early cardiotoxicity in patients undergoing HSCT.

Keywords: Cardiotoxicity, hematopoietic stem cell transplantation, right ventricle, tissue Doppler imaging, tricuspid annular plane systolic excursion

\section{INTRODUCTION}

Hematopoietic stem cell transplantation (HSCT) has become a mainstay in the treatment of various malignancies and benign conditions. ${ }^{[1]}$ The concept of high-dose (HD) chemotherapy plus HSCT has considerably improved clinical outcomes. Moreover, by reducing the intensity of conditioning regimens, the undesirable effects of allogeneic HSCT have been reduced, extending its indication to patients who were

Received: 18-01-2020 Revised: 25-02-2020 Accepted: 23-03-2020

Published Online: 11-06-2020

\begin{tabular}{|l|l|}
\hline \multicolumn{2}{c|}{ Access this article online } \\
\hline Quick Response Code: & Website: \\
& \\
http://www.ijcva.com
\end{tabular}

previously ineligible for the treatment. HD chemotherapy and conditioning regimens can result in rare but potentially hazardous cardiac complications. ${ }^{[2,3]}$ Hence, early evaluation of cardiac injury is of paramount relevance so that timely initiation of treatment may slow down or even stop disease progression. According to the current guidelines, cardiovascular evaluation

Address for correspondence: Dr. Cennet Yildiz, Kavram Meslek Yüksekokulu, Istanbul, Türkiye. E-mail: cennet_yildiz@live.com

This is an open access journal, and articles are distributed under the terms of the Creative Commons Attribution-NonCommercial-ShareAlike 4.0 License, which allows others to remix, tweak, and build upon the work non-commercially, as long as appropriate credit is given and the new creations are licensed under the identical terms.

For reprints contact: WKHLRPMedknow_reprints@wolterskluwer.com

How to cite this article: Tekiner F, Yildiz C, Guner SI. Evaluation of right ventricle functions might be a method for early recognizing cardiotoxicity in hematopoietic stem cell transplantation. Int J Cardiovasc Acad 2020;6:46-50. 
of HSCT patients involves annual measurement of lipid profile and fasting glucose levels in low-risk patients and echocardiographic and electrocardiographic examinations in symptomatic patients. Cardiac evaluation of patients is usually performed when clinically necessary ${ }^{[4]}$ However, by the time a diagnosis is made, complete recovery occurs in less than half of the patients. ${ }^{[5]}$ The timing of the cardiac assessment of HSCT patients without clinical symptoms is not well known, but routine screening and treatment may improve patient survival. ${ }^{[6]}$

Cardiac evaluation of oncology patients is usually performed with echocardiography, which is a quick, reliable, and noninvasive tool that can provide detailed physiological and morphological information about cardiac function. ${ }^{[7]}$ Most of the researches conducted so far on HSCT recipients have mainly focused on left ventricular structure and function. The right ventricle (RV) has a crescentic anatomical and morphological structure, making it difficult to evaluate its complex morphology and function. Tissue Doppler imaging (TDI) is an echocardiography mode that is based on the measurement of myocardial velocities. It enables us to quantitatively assess $\mathrm{RV}$ function throughout the cardiac cycle. ${ }^{[8]}$

To determine the impact of HSCT on RV function, conventional echocardiographic measurements and TDI were performed in 137 allogeneic or autologous HSCT patients before and after transplantation. The pre- and post-transplant values were compared in allogeneic and autologous recipients. Therefore, we aimed to evaluate RV function by sensitive techniques for the early recognition of cardiotoxic effects.

\section{Methods}

Patients with multiple myeloma, acute myeloid leukemia (AML), myelodysplastic syndrome, aplastic anemia, Hodgkin's lymphoma, and non-Hodgkin's lymphoma receiving allogeneic or autologous HSCT who underwent echocardiographic examination before transplantation were included in our study. Each patient gave informed consent before study enrollment. Approval of the study was obtained from the local ethics committee (approval number B 08.06 YOK 2.I.U.E.50.0.05.00/12, approval date: 12.04.2019). Our exclusion criteria were previous anthracycline use, inadequate echocardiographic images, hypertension, diabetes mellitus, a history of percutaneous coronary intervention or coronary artery bypass operation, arrhythmia, conduction disturbances, valvular heart disease (more than mild), and volume overload or depletion.

In total, 140 successive HSCT patients were studied during the study period. Three of the patients died before the second echocardiographic examination. The remaining 137 patients, who had both pre- and post-transplant examinations, were included in our study.

\section{Conditioning regimens}

After the patients were admitted to the bone marrow transplant (BMT) unit, stem cell mobilization was performed with etoposide plus granulocyte-colony-stimulating factor (G-CSF) in multiple myeloma and non-Hodgkin's lymphoma patients. Hodgkin's lymphoma patients achieved autologous stem cell mobilization with G-IVE (G-CSF, ifosfamide, VP-16, and epirubicin). The cells were collected by a cell separator unit (COB brand) on the $5^{\text {th }}$ day of mobilization. If the number of mononuclear cells was lower than $2.5-5 \times 10^{8} / \mathrm{kg}$ (median: $4 \times 10^{6} / \mathrm{kg}$ ), then a repeat apheresis was performed on the same day. After harvesting, the cells were kept frozen at $-198^{\circ} \mathrm{C}$, and induction chemotherapy was started. Lymphoma patients were conditioned with the BEAM regimen, which included carmustine, etoposide, cytarabine (ara-C), and melphalan. Multiple myeloma patients were treated with melphalan, and AML patients were treated with cyclophosphamide (2 days) followed by intravenous busulfan (1 day, 4 patients) or fludarabine (5 days, 6 patients). Patients with myelodysplastic syndromes and aplastic anemia were treated with cyclophosphamide, thymoglobulin (ATG), and fludarabine (4 days). The harvested cells were infused in patients 1 day after the completion of chemotherapy. The infusion was administered through the jugular or subclavian vein. G-CSF $5 \mathrm{mg} / \mathrm{kg}$ was started on the $5^{\text {th }}$ day. Control echocardiography was performed after approximately 2 weeks, which was when engraftment of the white blood cells occurred. Variables found in pre-BMT echocardiography were compared with the variables found in post-BMT echocardiography.

\section{Echocardiography}

Echocardiographic examinations of the patients were performed in the left lateral decubitus position with the use of a 2.5-3.5 MHz transducer (Vivid 7 system, GE-Vingmed, Horten, Norway). The same cardiologist who was specialized in echocardiography obtained the images from the standard parasternal, short-axis, and apical views. Pulsed Doppler recordings, standard M-mode, and two-dimensional and left ventricular ejection fraction (LVEF) measurements were performed according to the American Society of Echocardiography guidelines. ${ }^{[9]}$ Three measurements were made for each parameter and averaged. The first and second echocardiographic examinations were performed on the day before and 30 days after HSCT. Tricuspid annular plane systolic excursion (TAPSE) was measured at the lateral annulus of the tricuspid valve using an M-mode echocardiogram from an apical four-chamber view. ${ }^{[10]}$ The pulmonary artery systolic pressure was obtained as the sum of the right atrial pressure and trans-tricuspid gradient, which was estimated with the modified Bernoulli equation. ${ }^{[1]}$ The pulmonary acceleration time (PAT) was calculated from the onset to the peak velocity of the pulse Doppler pulmonary arterial velocity in the apical short-axis view. The ratio of PAT to the Pulmonary ejection time (PET) (PAT/PET) was calculated. ${ }^{[12]}$ TDI assessment of the RV was obtained at the lateral tricuspid annulus in an apical four-chamber view. ${ }^{[13]}$ To achieve a clear tissue signal, the gains were minimized, and the Nyquist limit was adjusted to $30 \mathrm{~cm} / \mathrm{s}$. The peak systolic 
velocity (S ') and early (E') and late (A') diastolic velocities were measured, and E'/A' was calculated.

The volume status of the patients was evaluated by measuring the inferior vena cava diameter from the subcostal inferior vena cava long-axis view within $2 \mathrm{~cm}$ of the right atrial junction. More than $50 \%$ inspiratory collapse of a normal-sized inferior vena cava $(1.5-2.5 \mathrm{~cm})$ was consistent with normal intravascular volume status.

\section{Statistical analysis}

The results of descriptive analyses were presented as means and standard deviations or median and interquartile range (range from the $25^{\text {th }}$ to the $75^{\text {th }}$ percentile). The categorical variables were expressed as numbers and percentages. Numerical variables were compared using the Student's $t$-test. The effect of age upon variables that showed $P<0.05$ was assessed by Pearson's correlation. Statistical analyses were performed using the SPSS software version 20 for Windows (SPSS Inc., Chicago, IL, USA), with $P<0.05$ considered statistically significant.

\section{RESULTS}

Table 1 illustrates the clinical characteristics of the patients. Among 137 HCST recipients, 86 had autologous graft, whereas 51 had allogeneic graft. Our study population comprised 41 autologous multiple myeloma, 25 autologous Hodgkin's lymphoma, 20 autologous and 19 allogeneic non-Hodgkin's lymphoma, 10 allogeneic AML, 12 allogeneic myelodysplastic syndrome, and 10 allogeneic aplastic anemia patients. All patients with multiple myeloma and Hodgkin's lymphoma received an autologous graft, and all patients with AML, myelodysplastic syndrome, and aplastic anemia had an allogeneic graft. The mean age of the patients at the time of transplantation was $46.51 \pm 16.24$ years, the mean hospital length of stay was 30 (ranged from 14 to 64) days, and the mean leukocyte engraftment and platelet engraftment time were 11 (ranged from 6 to 23) and 13 (ranged from 7 to 25) days, respectively. The number of female and male patients was $60(43.8 \%)$ and 77 (56.2\%), respectively.

There were no significant differences in left ventricular echocardiographic parameters including left ventricular end-diastolic diameter, left ventricular end-systolic diameter, LVEF, and E wave velocity, A wave velocity, E/A ratio, and Deceleration time (DT) before and after HSCT. When we analyzed the RV indices, we did not find any significant differences in RV, right atrium, pulmonary artery pressure, pulmonary velocity, PAT/PET, PAT, and A' velocity between pre- and post-HSCT groups. Compared to pretransplant values, posttransplant values of the E' velocity, S' velocity, E'/A' ratio, and TAPSE showed a statistically significant decrease $(0.15 \pm 0.34$ vs. $0.12 \pm 0.04, P=0.032 ; 0.19 \pm 0.06$ vs. $0.9 \pm 0.04, P<0.001 ; 0.90 \pm 0.40$ vs. $0.72 \pm 0.37, P=0.012$; and $2.30 \pm 0.39$ vs. $1.63 \pm 0.30, P<0.001$, respectively). Table 2 displays the echocardiographic parameters of the patients before and after HSCT.

\begin{tabular}{|c|c|}
\hline Characteristics & mean $\pm S D$ \\
\hline Age, years & $46.51 \pm 16.24$ \\
\hline \multicolumn{2}{|l|}{ Sex, $n(\%)$} \\
\hline Male & $77(56.2)$ \\
\hline Female & $60(43.8)$ \\
\hline Allogeneic graft, $n(\%)$ & $51(37.2)$ \\
\hline Autologous graft, $n(\%)$ & $86(62.8)$ \\
\hline \multicolumn{2}{|l|}{ Underlying disease, $n(\%)$} \\
\hline Multiple myeloma & $41(29.92)$ \\
\hline Hodgkin's lymphoma & $25(18.25)$ \\
\hline Non-Hodgkin’s lymphoma & $39(28.47)$ \\
\hline Acute myeloid leukemia & $10(7.30)$ \\
\hline Myelodysplastic syndrome & $12(8.76)$ \\
\hline Aplastic anemia & $10(7.30)$ \\
\hline
\end{tabular}
Table 2: Echocardiographic values of the patients
before (T1) and after (T2) hematopoietic stem cell
transplantation

\begin{tabular}{lccc}
\hline & T1 & T2 & $\boldsymbol{P}$ \\
\hline LVEDD, cm & $4.92 \pm 0.47$ & $4.88 \pm 0.53$ & 0.252 \\
LVESD, cm & $3.11 \pm 0.38$ & $3.13 \pm 0.47$ & 0.519 \\
LVEF, \% & $64.61 \pm 4.43$ & $64.30 \pm 5.00$ & 0.512 \\
E, m/s & $0.77 \pm 0.19$ & $0.76 \pm 0.18$ & 0.185 \\
A, m/s & $0.75 \pm 0.19$ & $0.78 \pm 0.24$ & 0.212 \\
E/A & $1.11 \pm 0.51$ & $0.98 \pm 0.36$ & 0.198 \\
DT, ms & $198.36 \pm 53.26$ & $196.25 \pm 49.64$ & 0.573 \\
RV, cm & $2.97 \pm 0.50$ & $2.96 \pm 0.47$ & 0.678 \\
RA, cm & $3.32 \pm 0.45$ & $3.30 \pm 0.32$ & 0.405 \\
PAP, mmHg & $21.43 \pm 6.40$ & $22.01 \pm 6.08$ & 0.231 \\
PV, m/s & $0.93 \pm 0.18$ & $0.94 \pm 0.17$ & 0.112 \\
PAT/PET & $0.43 \pm 0.06$ & $0.47 \pm 0.35$ & 0.221 \\
PAT, ms & $153.34 \pm 27.92$ & $152.56 \pm 24.88$ & 0.324 \\
E', m/s & $0.15 \pm 0.34$ & $0.12 \pm 0.04$ & 0.032 \\
A', m/s & $0.19 \pm 0.06$ & $0.19 \pm 0.68$ & 0.356 \\
S', m/s & $0.19 \pm 0.06$ & $0.09 \pm 0.04$ & $<0.001$ \\
E'/A' & $0.90 \pm 0.40$ & $0.72 \pm 0.37$ & 0.012 \\
TAPSE, cm & $2.30 \pm 0.39$ & $1.63 \pm 0.30$ & $<0.001$ \\
\hline
\end{tabular}

LVEDD: Left ventricular end-diastolic diameter, LVESD: Left ventricular end-systolic diameter, LVEF: Left ventricular EF, E: Mitral early diastolic velocity, A: Mitral late diastolic velocity, DT: Deceleration time, RV: Right ventricle, RA: Right atrium, PAP: Pulmonary artery pressure, PV: Pulmonary velocity, PAT: Pulmonary acceleration time, PET: Pulmonary ejection time, E': Tricuspid annular early diastolic velocity, A': Tricuspid annular late diastolic velocity, S': Tricuspid annular systolic velocity, TAPSE: Tricuspid annular plane systolic excursion

\section{Discussion}

In our study, we found that TAPSE, tricuspid annular S', E' velocities, and E'/A' ratio decreased significantly after HSCT. We found no significant differences with regard to LVEF and mitral diastolic velocities before and after HSCT.

After its first usage in 1980, HSCT has today become one of the most effective ways in treating various cancer types. ${ }^{[14]}$ As the number of HSCT patients has increased, cardiac complications 
have become more widespread that their prompt diagnosis is crucial in order to prevent permanent damage. HSCT can result in either acute or chronic cardiovascular complications. Acute cardiovascular complications are defined as those developing within 100 days of the treatment and include congestive heart failure (HF), cardiac tamponade, and arrhythmias, whereas chronic complications refer to the events that may occur even years after treatment. ${ }^{[15]}$ The pathogenesis of the myocardial damage after HSCT is related to direct toxic effects of the anticancer or conditioning treatment regimens ${ }^{[6]}$ Formation of reactive oxygen species and increased inflammatory activity have been accused of the underlying causes of congestive HF and coronary artery disease. ${ }^{[16-18]}$ Lifestyle modifications, physical inactivity, and graft versus host disease are some of the factors that are thought to contribute to an increased cardiovascular risk in HSCT patients. ${ }^{[19-21]}$ Anthracyclines by far are the most extensively studied drugs in terms of their cardiovascular adverse effects as well as treatment options and preventive strategies. Clinical features of the early and late anthracycline toxicity include left ventricular dysfunction, exercise intolerance, dyspnea, and HF symptoms, which are closely related to the anthracycline cumulative dose. ${ }^{[22]}$

Several studies have addressed the detection of subclinical cardiac damage in HSCT recipients. Patients with elevated levels of cTnI and BNP after HSCT may face a greater risk of mortality and HF in long-term care. ${ }^{[23-25]}$

Echocardiography is an easy, noninvasive, and widely used method that plays a key role in evaluating cardiac structure and function. Roziakova et al. ${ }^{[25]}$ evaluated left ventricular function, high-sensitive cardiac troponin $\mathrm{T}$ (hs-cTnT), and $\mathrm{N}$ terminal pro-B-type natriuretic peptide (NT-proBNP) levels in allogeneic HSCT patients. Both hs-cTnT and NT-proBNP levels begun to increase after the $1^{\text {st }}$ day of HSCT and persistently elevated at 1 month in about one-third of the patients. Moreover, LVEF and E/A ratio decreased in 13.5\% and $5.4 \%$ of patients, respectively, indicating newly developed systolic and diastolic dysfunction. In a study performed by Oliver et al., it has been shown that pro-BNP was the biomarker that increases during HSCT, and global longitudinal strain had the greatest specificity in predicting subclinical left ventricular dysfunction. In that study, $12.5 \%$ of patients had a significant reduction in Global longitudinal strain (GLS). ${ }^{[26]}$ Poreba et $a .^{[27]}$ have performed transthoracic echocardiography of 47 allogeneic and autologous HSCR patients before and at about 11 days after the procedure; in their study, LVEF, E/A, and E' were found to be decreased, whereas DT, Isovolumetric relaxation time (IVRT), and E/E' were found to be increased. Yet another study has shown that cardiac toxicity determined by a decrease in LVEF, developed $31 \%$ of the autologous HSCT recipients 18 days after transplantation. Cardiac toxicity occurred both with and without cTnI elevation, and the absence of anthracycline usage did not hinder the development of it. ${ }^{[28]}$ These results indicate that deterioration of left ventricular functions occurs as early as within 1 month after the treatment without clinical symptoms.
Previous studies have been predominantly based on the effects HSCT on left ventricular structure and function, but there has been little research about RV functions. The RV received little attention in these group patients. Hence, in our study, RV functions along with left ventricular functions were evaluated. In fact, RV function is one of the major determinants for survival in HF irrespective of the underlying etiology. ${ }^{[29]}$ In a study conducted to evaluate the effects of cancer chemotherapy on echocardiographic indices, it was found that RV systolic and diastolic functions were affected in a rather short period of time and earlier than the left ventricle. ${ }^{[30]}$ Moreover, LV dysfunction during cancer chemotherapy was associated with worsened RV functions.$^{[31]}$ Karvandi et al. showed that HSCT recipients had worsened diastolic function characterized by decreased tissue Doppler E' velocity in both the left and RV. ${ }^{[32]}$ An important aspect of TDI reveals itself in yielding an independent echocardiographic examination of the patient volume status, which is likely to change during HSCT. Although we found no significant differences in LV echocardiographic parameters, RV parameters such as TAPSE, S', E' velocities, and tricuspid annular mean E'/A' ratios decreased significantly in HSCT recipients, indicating earlier involvement of the RV. Chemotherapy-related cardiac toxicity primarily involves subendocardial layer of the heart. ${ }^{[33]}$ Because RV is thinner and contains less myofibrils compared to LV, it may be more vulnerable to the toxic effects of chemotherapeutics.

\section{Conclusion}

HSCT is associated with deteriorated RV systolic and diastolic functions. Moreover, RV functions seem to be affected earlier than the LV. TDI and TAPSE can be used as reliable measures to assess early cardiotoxicity in HSCT recipients.

\section{Limitations}

The limitations of our study are as follows: (1) as cTnI and BNP levels were not measured in our study, we do not know whether worsening of the RV functions was associated with a rise in cTnI and/or BNP concentrations; (2) patients were not followed up. It is not known what percentage of the patients who had subclinical cardiac dysfunction would have clinically relevant disease; and (3) we did not use strain and strain rate analysis for evaluation of the cardiac function.

\section{Financial support and sponsorship}

Nil.

\section{Conflicts of interest}

There are no conflicts of interest.

\section{REFERENCES}

1. Copelan EA. Hematopoietic stem-cell transplantation. N Engl J Med 2006;354:1813-26.

2. Hertenstein B, Stefanic M, Schmeiser T, Scholz M, Göller V, Clausen $\mathrm{M}$, et al. Cardiac toxicity of bone marrow transplantation: Predictive value of cardiologic evaluation before transplant. J Clin Oncol 1994;12:998-1004.

3. Fujimaki K, Maruta A, Yoshida M, Sakai R, Tanabe J, Koharazawa H, 
et al. Severe cardiac toxicity in hematological stem cell transplantation: Predictive value of reduced left ventricular ejection fraction. Bone Marrow Transplant 2001;27:307-10.

4. Majhail NS, Rizzo JD, Lee SJ, Aljurf M, Atsuta Y, Bonfim C, et al. Recommended screening and preventive practices for long-term survivors after hematopoietic cell transplantation. Hematol Oncol Stem Cell Ther 2012;5:1-30.

5. Armenian SH, Chow EJ. Cardiovascular disease in survivors of hematopoietic cell transplantation. Cancer 2014;120:469-79.

6. Scott JM, Armenian S, Giralt S, Moslehi J, Wang T, Jones LW. Cardiovascular disease following hematopoietic stem cell transplantation: Pathogenesis, detection, and the cardioprotective role of aerobic training. Crit Rev Oncol Hematol 2016;98:222-34.

7. Mookadam F, Sharma A, Lee HR, Northfelt DW. Intersection of cardiology and oncology clinical practices. Front Oncol 2014;4:259.

8. Bleeker GB, Steendijk P, Holman ER, Yu CM, Breithardt OA, Kaandorp TA, et al. Assessing right ventricular function: The role of echocardiography and complementary technologies. Heart 2006;92 Suppl 1:i19-26.

9. Lang RM, Bierig M, Devereux RB, Flachskampf FA, Foster E, Pellikka PA, et al. Recommendations for chamber quantification: A report from the American Society of Echocardiography's Guidelines and Standards Committee and the Chamber Quantification Writing Group, developed in conjunction with the European Association of Echocardiography, a branch of the European Society of Cardiology. J Am Soc Echocardiogr 2005;18:1440-63.

10. López-Candales A, Rajagopalan N, Gulyasy B, Edelman K, Bazaz R. Comparative echocardiographic analysis of mitral and tricuspid annular motion: Differences explained with proposed anatomic-structural correlates. Echocardiography 2007;24:353-9.

11. Schiller NB. Pulmonary artery pressure estimation by Doppler and two-dimensional echocardiography. Cardiol Clin 1990;8:277-87.

12. Chan KL, Currie PJ, Seward JB, Hagler DJ, Mair DD, Tajik AJ. Comparison of three Doppler ultrasound methods in the prediction of pulmonary artery pressure. J Am Coll Cardiol 1987;9:549-54.

13. Dokainish H, Abbey H, Gin K, Ramanathan K, Lee PK, Jue J. Usefulness of tissue Doppler imaging in the diagnosis and prognosis of acute right ventricular infarction with inferior wall acute left ventricular infarction. Am J Cardiol 2005;95:1039-42.

14. D'Souza A, Lee S, Zhu X, Pasquini M. Current use and trends in hematopoietic cell transplantation in the United States. Biol Blood Marrow Transplant 2017;23:1417-21.

15. Blaes A, Konety S, Hurley P. Cardiovascular complications of hematopoietic stem cell transplantation. Curr Treat Options Cardiovasc Med 2016;18:25.

16. Chen B, Peng X, Pentassuglia L, Lim CC, Sawyer DB. Molecular and cellular mechanisms of anthracycline cardiotoxicity. Cardiovasc Toxicol 2007;7:114-21.

17. Schultz-Hector S, Trott KR. Radiation-induced cardiovascular diseases: Is the epidemiologic evidence compatible with the radiobiologic data? Int J Radiat Oncol Biol Phys 2007;67:10-8.

18. Zhao W, Diz DI, Robbins ME. Oxidative damage pathways in relation to normal tissue injury. Br J Radiol 2007;80:S23-31.
19. Perhonen MA, Zuckerman JH, Levine BD. Deterioration of left ventricular chamber performance after bed rest: "cardiovascular deconditioning" or hypovolemia? Circulation 2001;103:1851-7.

20. Levine BD, Zuckerman JH, Pawelczyk JA. Cardiac atrophy after bed-rest deconditioning: A nonneural mechanism for orthostatic intolerance. Circulation 1997;96:517-25.

21. Tichelli A, Gratwohl A. Vascular endothelium as 'novel' target of graft-versus-host disease. Best practice and research. Clin Haematol 2008;21:139-48.

22. Shan K, Lincoff AM, Young JB. Anthracycline-induced cardiotoxicity. Ann Int Med 1996;125:47-58.

23. Snowden JA, Hill GR, Hunt P, Carnoutsos S, Spearing RL, Espiner E, et al. Assessment of cardiotoxicity during haemopoietic stem cell transplantation with plasma brain natriuretic peptide. Bone Marrow Transplant 2000;26:309-13.

24. Cardinale D, Sandri MT, Colombo A, Colombo N, Boeri M, Lamantia G, et al. Prognostic value of troponin I in cardiac risk stratification of cancer patients undergoing high-dose chemotherapy. Circulation 2004;109:2749-54.

25. Roziakova L, Bojtarova E, Mistrik M, Dubrava J, Gergel J, Lenkova N, et al. Serial measurements of cardiac biomarkers in patients after allogeneic hematopoietic stem cell transplantation. J Exp Clin Cancer Res 2012;31:13.

26. Oliver AC, Superchi R, Federico S, Matlide C, Silvana J, Errecarte C, et al. Role of echocardiography and cardiac biomarkers in early detection of hematopoietic stem cell transplantation associated cardiotoxicity. Blood 2019;134 Supp 1:5688.

27. Poręba M, Gać P, Usnarska-Zubkiewicz L, Pilecki W, Kuliczkowski K, Mazur G, et al. Echocardiographic evaluation of the early cardiotoxic effect of hematopoietic stem cell transplantation in patients with hematologic malignancies. Leuk Lymphoma 2016;57:2119-25.

28. Chung T, Lim WC, Sy R, Cunningham I, Trotman J, Kritharides L. Subacute cardiac toxicity following autologous haematopoietic stem cell transplantation in patients with normal cardiac function. Heart 2008;94:911-8.

29. Ghio S, Gavazzi A, Campana C, Inserra C, Klersy C, Sebastiani R, et al. Independent and additive prognostic value of right ventricular systolic function and pulmonary artery pressure in patients with chronic heart failure. J Am Coll Cardiol 2001;37:183-8.

30. Tanindi A, Demirci U, Tacoy G, Buyukberber S, Alsancak Y, Coskun $\mathrm{U}$, et al. Assessment of right ventricular functions during cancer chemotherapy. Eur J Echocardiogr 2011;12:834-40.

31. Calleja A, Poulin F, Khorolsky C, Shariat M, Bedard PL, Amir E, et al. Right ventricular dysfunction in patients experiencing cardiotoxicity during breast cancer therapy. J Oncol 2015;2015:609194.

32. Karvandi M, Piranfar MA, Yazdani S, Mehdizadeh M, Hajfathali A, Tabarraee M. Effect of bone marrow transplantation on diastolic function indices. Int J Clin Exp Med 2013;6:206-10.

33. Mortensen SA, Olsen HS, Baandrup U. Chronic anthracycline cardiotoxicity: Haemodynamic and histopathological manifestations suggesting a restrictive endomyocardial disease. $\mathrm{Br}$ Heart $\mathrm{J}$ $1986 ; 55: 274-82$. 